\title{
The Putamen Intensity Gradient in CJD Diagnosis
}

\author{
A. Hojjat ${ }^{1}$, D. Collie ${ }^{2}$, and A.C.F. Colchester ${ }^{1}$ \\ ${ }^{1}$ Medical Image Computing, Kent Institute of Medicine and Health Sciences, \\ University of Kent at Canterbury, UK \\ ${ }^{2}$ National CJD Surveillance Unit, Western General Hospital, Edinburgh, UK \\ a.colchester@ukc.ac.uk
}

\begin{abstract}
The deep grey matter structures of the brain have been reported to show MR hyperintensity in the sporadic form of Creutzfeldt-Jakob disease (sCJD), but the criteria for visual judgment of this are not well defined. We carried out a quantitative study of $\mathrm{T} 2$ weighted and proton density scans comparing 10 sCJD patients with 10 non-CJD dementia controls (NCD) and also with 11 patients suffering from the new variant form of CJD (vCJD). Scans were acquired in a clinical context and came from many hospitals. Absolute intensities varied widely and did not allow any useful discrimination. In all groups the putamen had a gradient of reducing intensity from anterior to posterior on T2 scans. In both s- and v- CJD patients this gradient was increased. Sensitivity and specificity (S\&S) for SCJD against NCD were $89 \%$. The T2 and PD intensities of the putamen relative to the other grey matter structures studied were not useful for distinguishing between any of the patient groups. The ratio of putamen to frontal white matter $\mathrm{T} 2$ intensity was significantly increased in vCJD compared to NCD and also to SCJD, while sCJD and NCD were indistinguishable by this test. We conclude that: (1) in our preliminary study, the putamen gradient appears to be important diagnostically for SCJD; (2) intensities of deep grey matter structures vary systematically and intensity-based segmentation methods used in patients and normals should take account of this.
\end{abstract}

\section{Introduction}

The deep grey matter structures of the cerebral hemispheres include the basal ganglia, which are mainly involved in motor control, and the thalamus, which is mainly involved in sensory processing. The basal ganglia include the caudate nucleus, the putamen and the globus pallidus. All these structures are anatomically distinct and are easily recognised in axial and coronal MR images of the brain. They are subject to a wide variety of acute and chronic pathological processes, which may selectively affect sub-parts of the deep grey structures, for example in Creutzfeldt Jakob disease (CJD) in which intensity abnormalities have been reported $[9,11]$. There is strong evidence from visual analysis that increased signal in the posterior part of the thalamus may be diagnostic for the new variant form of CJD (vCJD) $[1,3,4,8]$. There is less clear evidence that increased signal in the caudate nucleus and/ or the putamen may be diagnostic for the classical sporadic form of CJD (sCJD) $[2,5]$.

We have been using quantitative analyses to validate and extend these observations [1]. For a particular structure, absolute MR intensities obtained using a particular sequence vary widely according to several poorly controlled parameters, even for 
"standard" sequences (e.g. "T2 weighted"), so it is difficult to compare data from different scanners. Thus, for a rare disease like CJD, when data are often acquired at different hospitals using different scanner manufacturers and different settings, it is notable that visual analyses indicate that significant intensity differences are still identifiable. A central issue in quantitative analysis is how to define a normalisation method to reduce the variability of intensity estimates within diagnostic patient groups, while preserving or increasing the variability between diagnostic groups. The most obvious approach is to identify in the same scan specific reference structures against which the abnormal structures can be compared. For variant CJD we have recently evaluated different reference structures and shown that very high sensitivity and specificity for vCJD diagnosis can be achieved when MR examinations from a wide variety of scanners are analysed [1].

In the present work we examined putamen intensity changes using different normalisation approaches. It has been noted that intensity appears to reduce gradually from front to back on T2 axial slices [2]. Systematic study of putamen T2 intensities thus requires either (1) averaging the whole putamen cross-section, or (2) division of the putamen into reproducible sub-parts within which the intensities are recorded, or (3) quantifying the spatial rate of intensity change within the putamen. We evaluated these approaches in the context of CJD diagnosis. The results have relevance not only to this clinical condition but also to a range of other MR applications including intensity-based tissue class segmentation.
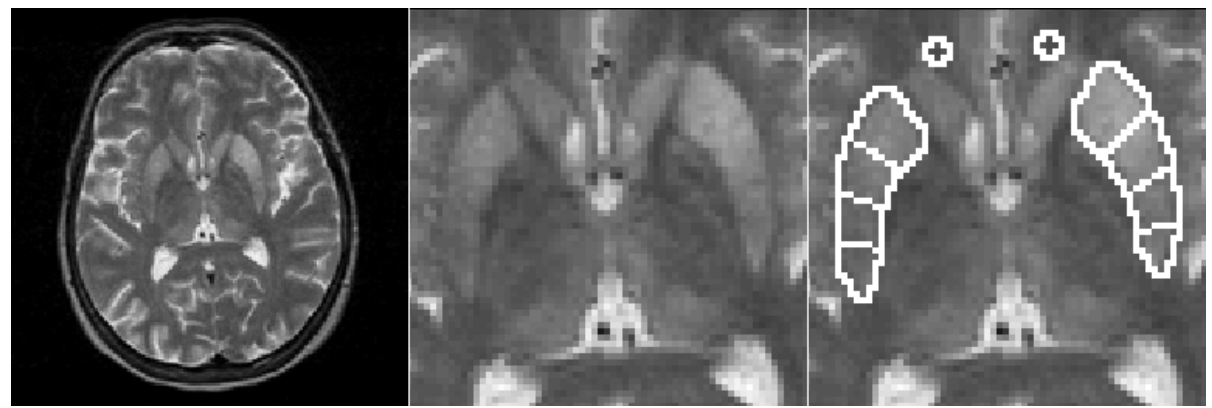

Fig. 1. (Left) T2-weighted axial MR slice through level of the putamen. (Middle) Magnified view of region containing the putamen. (Right) Outline shows putamen (segmented into 4 segments) and frontal white matter region

\section{Methods}

Dual echo (simultaneously acquired PD and T2-weighted) scans from three groups of patients with (1) sporadic CJD, (2) variant CJD and (3) non-CJD dementia (NCD) were studied. Prior to any intensity analyses, all available scans were graded for quality by visual inspection on two predefined 5-point subjective scales ( 5 being top quality), one scale for the level of contrast/noise and the other one for freedom-frommovement-artefact. Of a total of 73 scans, 32 scans were rejected as being very poor quality (graded 1 or 2 on either scale). This rejection rate reflects the fact that scans were obtained in clinical circumstances from a variety of hospitals, in patients who were often confused and uncooperative. Two additional scans were rejected because 
there was a focal lesion such as a lacunar infarct visible in one putamen. The remaining 39 scans (all scoring at 3 or greater on both scales) were included in the quantitative analyses.

There were 12 scans from 10 patients aged 50-76 with sporadic CJD, 11 scans from 11 patients aged 17-36 with variant CJD, and 16 scans from 10 patients aged 1476 with dementia in whom CJD had been excluded. All patients had dual echo, fast spin-echo MR scans with repetition time values ranging from 2862 to $4200 \mathrm{msec}$, and echo time values from $12-22 \mathrm{msec}$ for PD and from $80-120 \mathrm{msec}$ for T2 scans. Slice thickness ranged from 3 to $5 \mathrm{~mm}$. Data were all manipulated digitally; no hard copies were used.

Partial volume error (PVE) is an important potential confounding factor in any intensity analysis. The height of the putamen, in the cranio-caudal direction, is about $20 \mathrm{~mm}$ for much of its antero-posterior extent. We decided to restrict the present work to analysis of the axial (i.e. approximately parallel to the anterior commissureposterior commissure line) which passed through the putamen at mid-height (c.f. Fig 1 , left). This meant that most or all of the observed antero-posterior gradation of T2 intensity could not be a result of PVE.

In each scan the putamen and a region of interest in the frontal white matter were segmented interactively by a trained observer who viewed both the T2 and corresponding PD slice on the same display. The PD and T2 images were linked so that each anatomical structure could be outlined using either or both of the two images. In order to study the gradation of intensity, the putamen was subdivided into four parts of similar antero-posterior length, Fig 1 (middle). For each segment the average intensity was calculated and normalised by expressing intensities as ratios of different possible reference structures. Considering the putamen segments starting at the front, the ratio of the first and second part was similar to the ratio of the second and third part. We therefore fitted a straight line to the log transformed intensity ratios and examined the gradient of this line.

For each of the four putamen segments, the T2 and PD indices (raw values, normalised values etc) obtained from different patients were plotted. There was a strong linear correlation between T2 and PD values. To evaluate the simultaneous use of T2 and PD values in discriminating between patient groups, a straight line was fitted to the data on the scatter plot, and a line perpendicular to this was used as a threshold. Sensitivities and specificities using different thresholds were then calculated. These results were plotted as receiver operator characteristic (ROC) curves which showed the performance of the index in discriminating between SCJD and NCD, vCJD and NCD, and between SCJD and VCJD. One ROC curve plotted the true positive fraction (number of correctly categorised patients divided by the total number of patients with this diagnosis) versus the false positive fraction (number of incorrectly categorised control patients divided by the total number of these controls) [6]. The true positive fraction (TPF) is sensitivity and false positive fraction (FPF) is (1- specificity). Each ROC curve showed how sensitivity and specificity varied according to different thresholds. It is important to note that the optimum threshold selected for a diagnostic test depends on the clinical circumstances in which the test is being used, which may require high sensitivity at the expense of some loss of specificity, or high specificity at the expense of some loss of sensitivity. The ROC curve shows this pay-off graphically. One important point on the ROC curve is where the sensitivity and specificity are equal, and the value at this point was noted as a simple way to compare different indices. 


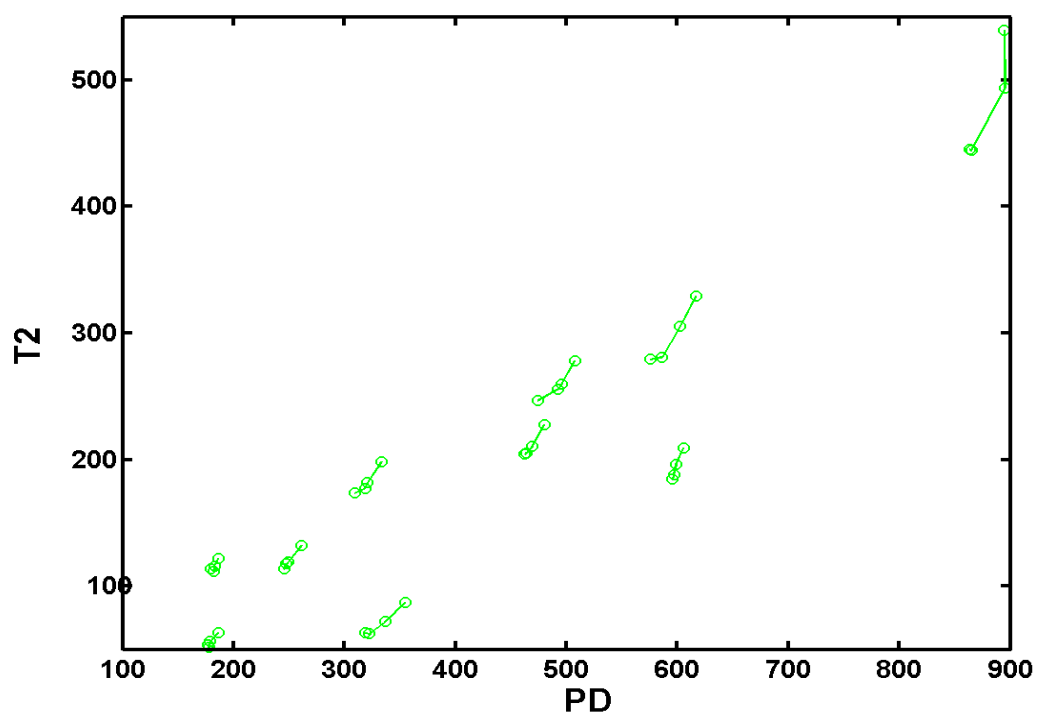

Fig. 2. Scatter plot of absolute T2 and PD values in four segments of the putamen in the vCJD group. Values of consecutive segments from one patient (corresponding right and left segments having been averaged) are connected by a line

\section{Results}

As expected, the absolute values of $\mathrm{T} 2$ and $\mathrm{PD}$ intensities for the putamen varied very widely between patients (T2 range 50-600; PD range 180-950). The range of variation within one putamen was very small compared to this (T2 range 15-150; PD range 20-120). Fig 2 shows the putamen values for the vCJD clinical group. The anterior three segments of the putamen showed progressive reduction of T2 intensity from front to back in nearly all patients (c.f. Fig 2). The posterior segment was more variable and subsequent analyses mainly focused on the front three parts.

Indices based on the ratio of putamen intensity divided by the frontal white matter intensity were examined. Results were similar whether the whole putamen or specific segments were used. Fig 3 (left) shows the scatter plot of the log of this index calculated for the whole putamen, averaged left and right, for T2 and for PD, with different clinical groups identified by different symbols. Fig 3 (left) shows that PD values did not help to discriminate between groups. However, for $\mathrm{T} 2$ there was hyperintensity of the putamen relative to frontal white matter in VCJD patients allowing good discrimination from both NCD controls and from SCJD patients, but SCJD patients were indistinguishable from NCD. For a particular discrimination - e.g. sCJD versus nonCJD dementia, the effect of using a chosen threshold for this index on the T2 axis can readily be visualised, and quantified by calculating the TPF (the proportion of SCJD patients correctly categorised) and FPF, (the proportion of non-CJD dementia patients incorrectly categorised as sCJD). The line $\mathrm{k} 1=1.25$ in Fig 3 (left) is the threshold value for the $\mathrm{T} 2$ index which generates approximately equal sensitivity and specificity for sCJD against control group. For a full range of possible thresholds, the TPF and FPF values can be plotted as an ROC curve (Fig 3 (right)). 
ROC curves derived from a limited number of data points are jagged (curve 1 in Fig 4 (left)). We chose to smooth the raw ROC curve using five iterations of a simple 3-point filter rather than fit a parametric function [6]. The resulting curves (both curves in Fig 3 (right) and curve 2 in Fig 4 (left)) follow the trend of the data closely and allow simple interpolation.
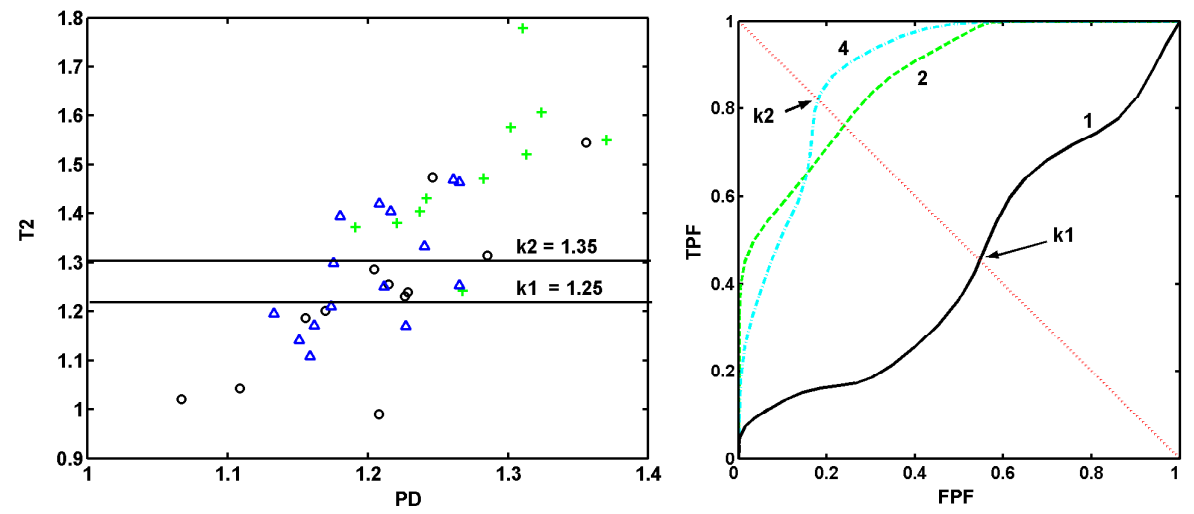

Fig. 3. (Left) Scatter plot of log intensity ratios (averaged left and right) for whole putamen divided by frontal white matter $(\mathrm{FWM})$ in T2 versus PD. Circles = sCJD; pluses = vCJD; triangles $=$ non-CJD dementia $(\mathrm{NCD})$ controls. Horizontal lines: examples of $\mathrm{T} 2$ thresholds which generate approximately equal sensitivity and specificity: k1 for SCJD vs NCD and k2 for SCJD vs vCJD. (Right) ROC curve for T2 log intensity ratios (averaged left and right), for whole putamen divided by frontal white matter (FWM). TPF $=$ true positive fraction (or sensitivity); FPF $=$ false positive fraction (or $1-$ specificity). Curve $1=\operatorname{sCJD}$ vs NCD; curve $2=$ vCJD vs NCD; curve $4=$ vCJD vs SCJD

Various methods of estimating rate of reduction of intensity along the putamen (anterior to posterior segments) were examined and gave good discriminatory results. Fig 4 (left) shows the ROC curve for the following method. For each patient the intensity of the second most anterior putamen segment was divided by the anterior putamen segment, for the right side and the left side separately. The smaller of the log ratios (corresponding to the steeper gradient) was noted for this patient. Very good discrimination of SCJD from NCD was achieved using this method, with the sensitivity and specificity balanced at approximately $89 \%$.

The ROC curves show how the TPF and FPF vary with respect to each other as the threshold is changed, but they do not show what specific thresholds generated which points on the ROC curve. We therefore show this mapping explicitly in Fig 4 (right) for the same data that are shown in Fig 4 (left). The log threshold ratio is plotted on the horizontal axis, against the sensitivity and specificity.

\section{Discussion}

Following published reports that putamen MRI signal intensities are high in SCJD, we expected normalisation using white matter (which has not been observed to show MR abnormalities in CJD) to provide reasonable discrimination between SCJD and nonCJD dementia (NCD) controls. We could not verify this quantitatively. However, 
we were able to demonstrate a clear difference in intensity in vCJD patients and this meant that putamen intensities normalised in this way could be used to discriminate with high confidence between vCJD and SCJD in our patients.

Within the putamen, the T2 intensity is varies systematically. We observed that the rate of reduction of $\log \mathrm{T} 2$ intensity of the putamen from front to back was approximately linear in all patient groups. The within-structure changes allowed us to normalise one part of the putamen with respect to another, with impressive results for discriminating SCJD patients from NCD. Our results were achieved without the use of bias field correction techniques; normalisation using nearby structures will reduce the effect of intensity inhomogeneity artifacts on the normalised indices.

Our approach allows us to select a different balance of sensitivity and specificity by varying the threshold. For diagnosis of sCJD from the T2 ratio mid-anterior / anterior putamen (Figs 4), we balance sensitivity and specificity at a ratio threshold of 0.93. However, in a clinical context such as screening a large group of patients with the goal of picking up almost all sCJD cases, Fig 4 (right) shows that the ratio threshold can be increased to 0.95 (e.g. an intensity change over 5\% is labeled as abnormal) in order to increase sensitivity to $99 \%$, at the cost of reducing of specificity to about $75 \%$. In contrast, at times one may require a very high specificity, for example in a context where a risky but potentially curative treatment is under consideration. While the specificity of our test could be increased to $99 \%$ by reducing the threshold to 0.89 (e.g. $11 \%$ intensity change), this would be at a cost of a substantial fall in sensitivity to about $39 \%$, which would probably be unacceptable.

An important group of methods for MR brain segmentation depend on the differences in MR signal intensity between grey matter, white matter and cerebrospinal fluid. These methods usually attempt to classify the deep grey matter structures into a single class of voxels. The present paper has drawn attention to the systematic and wide variation of intensity which may occur within a single grey matter structure. This variation increases errors when using current intensity-based segmentation methods. Future work should consider how modeling such variation could be used to improve accuracy.

\section{Conclusions}

1. Nearly all discriminatory diagnostic information came from T2 weighted images. However, if co-registered PD scans are available (usually this implies dual echo acquisition), some increased confidence in diagnosis can be achieved.

2. Quantitative processing allowed intensities to be measured reproducibly. While visual inspection is good for judging which of the two structures is the brighter, computer processing can provide additional information, for example allowing non-unity ratios to be quantified. Another benefit was that the threshold used for discrimination can be varied, to prioritise sensitivity or specificity for different applications. Plotting the sensitivity and specificity against the threshold contained all the information in a traditional ROC curve and had some advantages.

3. Putamen intensities normalised to a frontal white matter region do not discriminate between SCJD and non-CJD dementia (NCD) controls. However, a putamen/ frontal white matter intensity ratio of 1.35 or greater was a useful test for distinguishing between SCJD and vCJD (82\% sensitivity and specificity) and a ratio of 1.4 or greater had $78 \%$ sensitivity and specificity for vCJD against NCD. 

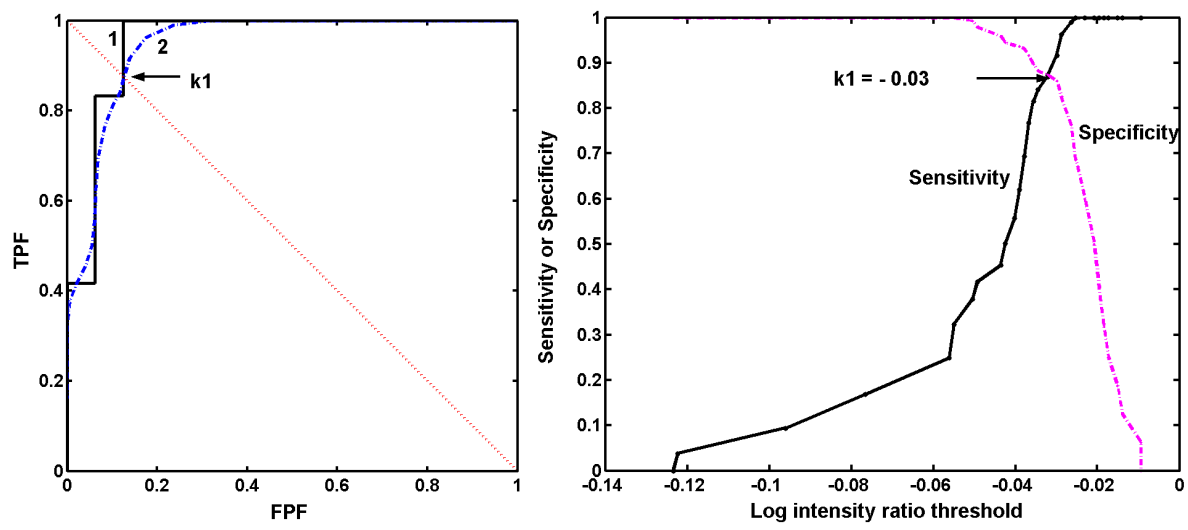

Fig. 4. (Left) ROC curve for diagnosis of SCJD versus non-CJD dementia using log T2 intensity ratios of mid-anterior putamen divided by anterior putamen (minimum of right ratio or or left ratio). TPF $=$ true positive fraction (sensitivity); FPF $=$ false positive fraction $(1-$ specificity). Curve 1 = raw data; curve $2=$ smoothed ROC curve. The smoothed ROC curve data and point $\mathrm{k}$ are also used to generate the right figure. (Right) Graph showing log intensity ratio threshold (horizontal axis) versus sensitivity and specificity. The threshold $\mathrm{k}=-0.03$ is a putamen gradient of 0.93 (i.e. $7 \%$ reduction in intensity from the anterior segment to the second segment along the putamen)

4. The rate of change of intensity along the putamen (the putamen gradient) on T2 scans was higher in SCJD and in vCJD than in non-CJD dementia controls. A gradient of 0.93 (i.e. a fall of $7 \%$ ) or greater between one segment and another along the putamen has high sensitivity and specificity for diagnosis (89\%).

5. Differences between hemispheres were slight and the ratio of indices for one hemisphere divided by those for the other were of no value in differential diagnosis.

\section{Acknowledgements}

This work was supported by the EU-funded project "Quantitative Analysis of MR scans in CJD (QAMRIC)" BMH 4-98-6048. The authors are grateful to Emily Colchester who carried out the interactive segmentation of anatomical structures.

\section{References}

1. Colchester, A. C. F., Hojjat, S. A., Will, R. G., and Collie, D. A. Quantitative validation of MR intensity abnormalities in variant CJD. Journal of Neurology, Neurosurgery of Psychiatry, in Press, 2002.

2. Collie, D. A., Sellar, R. J., Zeidler, M., Colchester, A. C. F., Knight, R., and Will, R. G. MRI of Creutzfeldt-Jakob disease: imaging features and recommended MRI protocol. Clinical Radiology 56, 726-739. 2001.

3. Collie, D. A., Sellar, R. J., Ironside, J., and et al. New variant Creutzfeldt-Jakob disease: diagnostic features on MR imaging with histopathologic correlation. Proceedings 36th Meeting of American Society of Neuroradiology , 139. 1998. 
4. Coulthard, A., Hall, K., English, P. T., and et al. Quantitative analysis of MRI signal intensity in new variant Creutzfeldt-Jakob disease. British Journal of Radiology 72, 742-748. 1999.

5. Finkenstaedt, M., Szudra, A., Zerr, I., Poser, S., Hise, J. H., Stoenbner, J. M., and Weber, T. MR Imaging of Creutzfeldt-Jakob disease. Radiology 199, 793-798. 1996.

6. Metz, C. E., ROC methology in radiologic imaging, 1986, Invest. Radiol. 21, 720-733.

7. Uchino, A., Yoshinaga, M., Shiokawa, O., Hata, H., and Ohno, M. Serial MR imaging in Creutzfeldt-Jakob disease. Neuroradiology 33, 364-367. 1991.

8. Zeidler, M., Sellar, R., Collie, D. A., Knight, R., Stewart, G. E., Macleod, M-A., Ironside, J., Cousens, S. N., Colchester, A. C. F., Hadley, D. M., and Will, R. G. The pulvinar sign on magnetic resonance imaging in variant Creutzfeldt-Jakob disease. Lancet, 355(9213):1412-1418, April 2000. 